\title{
ÜBERLEGUNGEN ZU DISKURSIVEN PRAXEN ÜBER ÖSTLICHES EUROPA UND DEUTSCHSPRACHIGE MINDERHEITEN IM SLOWENISCHEN RAUM
}

\section{EINLEITUNG}

Sucht man für das Syntagma östliches Europa Übersetzungsäquivalente im Slowenischen, stößt man bald auf Schwierigkeiten, die auch referenzieller Art sind: Handelt es sich bei deutschen Sprachminderheiten im östlichen Europa um „,nemške jezikovne manjšine v vzhodni Evropi“, „,nemške jezikovne manjšine v Vzhodni Evropi““ oder aber um ,nemške jezikovne manjšine vzhodnoevropske regije“"? ${ }^{1}$ Dieses Dilemma scheint bei Mitteleuropa etwas weniger ausgeprägt zu sein. Dabei reflektiert man den Begriff bekanntlich als Erkenntniskategorie und Raum-Modell (Földes 2011), der kulturell, sprachlich, politisch, sozialhistorisch traditionell mehrfach kodiert ist. Dies kommt bei östlichem Europa auf Anhieb weniger evident zum Vorschein, zumal dazu auch referenzsemantisch überschneidende Kodierungen - auch in der germanistischen Fachkommunikation, besonders im letzten Dezennium - durch Begriffe wie Ostmitteleuropa, ostmitteleuropäisches Areal, (Ost-)Mitteleuropa, Südosteuropa kursieren. Zieht man zum Verständnis des Begriffs östliches Europa Erklärungen aus weiteren Fachdisziplinen heran, so lassen sich spezifische Antworten eruieren, wie etwa in der Geografie, wo unter Osteuropa bestimmte Staaten aufgezählt werden und östliches Europa überhaupt nicht verwendet wird. Hingegen werden aus bildungspolitischer Sicht zu Regionen des östlichen Europa das Baltikum, Mittelosteuropa, die GUS-Staaten und Südosturopa gezählt. ${ }^{2}$ In der Germanistik wird wiederum anders differenziert, wobei das Gemeinte mit dem ,hic et nunc“ des Beobachters zusammenhängt. Bei der Definierung des östlichen Europa werden Kategorien wie Index, Zentrum, Beobachtungspunkt, Sprecherposition bemüht, während für einen slowenischen Germanisten dieses Syntagma etwa für den Raum von Ljubljana (oder vielleicht auch Murska Sobota) bis nach Russland, Griechenland und dem Baltikum steht, und für einen österreichischen

Ursula.KrevsBirk@ff.uni-lj.si

1 Kontrastivlinguistisch betrachtet handelt es sich um drei fast identische Übersetzungsäquivalente des Syntagmas deutsche Sprachminderheiten im östlichen Europa. Die Groß- bzw. Kleinschreibung bei Vzhodna Evropa und vzhodna Evropa soll der Feindifferenzierung zwischen der onymischen und (teil)appellativischen Verwendung dienen (was allerdings für Verwirrung bei der Befolgung dieser orthografischen Vorschrift sorgt): $v$ Vzhodni Evropi ,in Osteuropa“ (onymisch) vs. v vzhodni Evropi ,in Osteuropa ‘ (appellativisch); mit dem Übersetzungsäquivalent $v$ vzhodnoevropskih regijah (wortwörtlich ,in Regionen im Osten Europas') schafft man wiederum mehr Interpretationsraum.

2 https://osteuropa.lpb-bw.de/ (12.09.2020). 
Germanisten ist dieser Raum möglicherweise jenseits der östlichen Staatsgrenze Österreichs zu bestimmen.

Die Bezeichnung östliches Europa ist sicherlich symptomatisch für den Standpunkt, der hier nicht zufällig ausfallen kann. Er wird anfänglich auf den slowenischen Raum bezogen, der durch seine polyvalente Verortung als mitteleuropäischer und gegebenenfalls zentraleuropäischer, ferner auch südosteuropäischer, südslawischer, balkanstaatlicher und ex-jugoslawischer, nicht selten auch als ein erst seit 1991 existenter Raum, perzipiert wird, da er lediglich oder vordergründig mit dem slowenischen Staat assoziiert wird. Seine geographische Lage als slowenischsprachiger Raum mit Überschneidungsgebieten befindet sich hingegen offensichtlich am südlichen Rand des zusammenhängenden deutschen Sprachraums, am westlichen ungarischen Rand, am östlichen Rand der italienischen und friaulischen Sprachregionen und im Norden der südslawischen Sprachräume.

Vor diesem Hintergrund ist das Ziel des vorliegenden Beitrages ${ }^{3}$, einige Aspekte des Diskurses über deutsche Sprachminderheiten und Mehrsprachigkeit, besonders im slowenischen Raum, aber auch darüber hinaus, zu beleuchten. Diese Überlegungen, die auf Beobachtungen von medialisierten Äußerungen verschiedener Akteure beruhen, sind als Prolegomena zum Themenfeld deutschsprachige Minderheiten in Slowenien und ihren lebensweltlichen Referenzen zu verstehen. Medialisierte Äußerungen, die als solche in und von der Gesellschaft wahrgenommen werden oder wahrgenommen werden sollten, werden in Anlehnung an Foucault (2008) als Diskurs verstanden. Hierbei wird versucht, auf einige diskursive Praxen ${ }^{4}$ und somit auf einige Fragen der Minderheits- und Mehrsprachigkeitsproblematik hinzuweisen wie auch auf die Notwendigkeit weiterer Diskussionen. Dies sollen einige Beispiele aus dem aktuellen Sprachminderheitendiskurs veranschaulichen.

\section{2 ÖSTLICHES EUROPA UND MEHRSPRACHIGKEIT MIT DEUTSCH}

Das Syntagma östliches Europa ist nicht nur Bestandteil von Raummodellierungen innerhalb verschiedener Disziplinen, wie einleitend angedeutet, sondern auch ein Konzept im eigentlichen Sinne. Als Beispiel sei hier auf den Aufgabenbereich des Forschungszentrums „Deutsch in Mittel-, Ost- und Südosteuropa“ (FZ DiMOS) an der Universität Regensburg verwiesen, wo in der Vorstellung dieser Einrichtung auf der Homepage folgende Positionierung angekündigt wird: „Das FZ DiMOS sieht es als seine vornehmliche

3 Der Beitrag entstand im Rahmen der von der slowenischen Forschungsagentur ARRS geförderten Programmgruppe Theoretische und applikative Sprachforschungen: kontrastive, synchrone und diachrone Aspekte./Teoretične in aplikativne raziskave jezikov: kontrastivni, sinhroni in diahroni vidiki - P6-0218 (A) sowie des Forschungsprojektes Migrationsbedingte Mehrsprachigkeit mit Deutsch in Slowenien und Deutschland - sprachbiografische Untersuchungen (FZ DiMOS, SLO-1). An dieser Stelle sei dem DAAD für die Ermöglichung des Forschungsaufenthaltes an der Universität Regensburg ausdrücklich gedankt.

4 Inhaltlich werden damit medialisierte Aussagen in Form sprachlicher Realisierungen (Behauptungen) erfasst, die an bestimmte Institutionen, ihre Träger und Einzelautoren gebunden sind, und die zu einem bestimmten Zeitpunkt medialisiert wurden. Damit fungieren diese Aussagen als sozialer Akt einer Wirklichkeits(re)konstruktion. 
Aufgabe, die deutsche Sprache im östlichen Europa (hervorgeh. v. U. K. B.) im Rahmen der historischen und aktuellen Mehrsprachigkeitssituation dieses Raumes und in enger Einbeziehung der dortigen Nachbarsprachen des Deutschen und dortiger universitärer und anderer wissenschaftlicher Einrichtungen zu erforschen und zu dokumentieren. “" Die sich anbietende Zusammenfassung von Mittel-, Ost- und Südosteuropa durch östliches Europa bezieht sich nicht nur auf einen relativ breit gefassten Raum, der durchaus geografisch und kulturell gedeutet wird und zu dem auch Mitteleuropa gezählt wird, sondern es werden zugleich auch Fragenstellungen aufgegriffen, die in einem bestimmten - östlichen - Raum von identischer oder vergleichbarer Art relevant sind. Diese werden in den Leitlinien des FZ DiMOS zwar nicht näher erklärt, allerdings wird unter dem historischen und aktuellen Aspekt auf das Leitthema des Diskurses - die deutsche Sprache fokussiert. Dem Deutschen, das geschichtlich betrachtet eine Herrschersprache war, wird nach wie vor eine historisch gewachsene mehrfach kodierte Kommunikationsfunktion zugeschrieben, was neue oder zusätzliche Funktionszuschreibungen nicht ausschließt: „Ideelle Leitlinie ist es dabei, das Deutsche nicht mehr als Herrschaftssprache oder Nationalsprache zu begreifen, sondern als Interregionalsprache und als Sprachklammer in Mittel-, Ost- und Südosteuropa als Raum jahrhundertelanger, auch aktueller Migrationsbewegungen." Durch diese definitorische Neupositionierung der deutschen Sprache aus der Sicht ihrer Vermittlungsfunktion (interregionale Sprache, Sprachklammer) im besagten Raum und im Kontext historischer und aktueller Migration und Mehrsprachigkeit wird die Perspektive auf das Deutsche erweitert. So wird Deutsch als derjenige Faktor definiert, der, wie im Weiteren noch gezeigt werden soll, in diversen Lebens- und Sprachkonstellationen für Angehörige der Sprachminderheiten nicht nur politisch und kulturell, sondern auch psychosozial relevant ist und auf kollektiver und individueller Ebene ihre Mehrsprachigkeit mitkonstituiert.

Deutsch ist im heutigen Europa als ein konstitutiver Teil zahlreicher Mehrsprachigkeitssituationen interpretierbar, die vielfältige Konstellationen von Sprachkontakten erkennen lassen. Diese Kontaktvielfalt ist in Mittel- bzw. Ost- und Südosteuropa auf eine über mehrere Jahrhunderte andauernde Koexistenz der einzelnen Sprachen mit dem Deutschen zurückzuführen. Sprachgeschichtliche und kulturgeschichtliche (Sonder)Entwicklungen innerhalb dieser Kulturräume und ihrer Sprachen sind weiterhin wirksam und lassen sich auch in der Gegenwart ausmachen. Sie sind für Interaktionsbeziehungen zu Nachbarsprachen und -kulturen wie auch für aktuelle weiträumige Migrationsprozesse in spezifischer Weise bestimmend und bedingen somit auch das Sprachverhalten mehrsprachiger Individuen.

Obwohl Deutsch außerhalb des geschlossenen Sprachraumes in Mittel- und Südosteuropa oft als historisch gewachsene Sprache begriffen wird, wird ihr gegenwärtig vermehrt die Funktion eines referenziellen Kulturidioms zugeschrieben, das den europäischen Sprachbund weitgehend mitbestimmt, da es nach Kurzová (1996) mit weiteren Fokussprachen Tschechisch, Slowakisch und Ungarisch und den Marginalsprachen Polnisch und Slowenisch das Sprachareal Mitteleuropas bestimmt. Als sein „zentrales

5 https://www.uni-regensburg.de/forschung/dimos/startseite/index.html (15.05.2020). 
Kontaktareal“" in Mitteleuropa (Newerkla 2017: 20) wird das Kontaktareal Altösterreich betrachtet, ,in dem das Deutsche als Lingua franca bzw. als Verkehrssprache (zumindest in den Städten) protegiert wurde.“ (ebd.). In diesem Kontaktareal befinden sich aufgrund der Konvergenzen das österreichische Deutsch von Wiener Prägung und weitere Sprachen der Habsburgermonarchie, d. h. nicht nur Ungarisch, Tschechisch, Slowakisch oder Polnisch und Slowenisch, sondern ,jedenfalls auch das Kroatische, das auf damals österreichischem Boden gesprochene Serbische, Ukrainische und Rumänische sowie für einige Zeit auch das Bosnische" (ebd.: 21). Durch die Berücksichtigung der erwähnten slawischen Sprachen wie auch des Ukrainischen und Rumänischen weitet sich das - mitteleuropäische - Kontaktareal Altösterreich in das östliche Europa aus, in dem Deutsch zunehmend seinen Status als interregionale Sprache festigt und dadurch in entscheidender Weise zur Intensivierung und Pluralisierung von bilateralen und multilateralen Sprach- und Kulturkontakten beiträgt.

Die Fokussierung auf Deutsch ist in diesem Zusammenhang folglich notwendig und auch berechtigt, da der slowenische Sprach- und Kulturraum in seiner Geschichte eng mit dem deutschen verbunden war und unter mehreren Aspekten auch weiterhin ist. Im slowenischen Raum nimmt Deutsch aus historischer soziokultureller oder soziolinguistischer Perspektive eine Sonderstellung ein. Dazu einige Eckdaten: Die Nachbarsprachen Deutsch und Slowenisch sind Kontaktsprachen, die bis 1918 im Staatsrahmen der k. u. k. Monarchie auf dem slowenischen ethnischen Gebiet sogar koexistent waren und im zweisprachigen Gebiet im österreichischen Südkärnten und teilweise in der Steiermark an der österreichisch-slowenischen Grenze auch nach wie vor sind. Deutsch war bis Mitte des 19. Jahrhunderts auch die dominante Sprache, an der die slowenische Kultur ausgerichtet war. Bis zur Etablierung und Standardisierung des Slowenischen war Deutsch als Amtssprache vorherrschend und als Kulturidiom auch ausschlaggebend für das Slowenische, und zwar bis zur zweiten Hälfte des 19. Jahrhunderts. Viele Kulturschaffende wie etwa France Prešeren oder Jernej Kopitar waren bilingual und verfassten ihre Werke in beiden Sprachen. Die historische Mehrsprachigkeit und die vielfältigen Kontakteinwirkungen hinterließen Spuren in beiden Sprachen, in Kontaktregionen des österreichischen Deutsch (Pohl 2019 u. a.) ${ }^{6}$ einerseits und in sämtlichen Varietäten des Slowenischen ${ }^{7}$ (vgl. Šekli 2015, Javor Briški 2020) andererseits, wobei der deutschsprachige Einfluss auf das Slowenische vorherrschend war. Wie stark die historische Einwirkung des diskursiv und kommunikativ dominanten Deutschen auf die (diskursiv) schwächeren Kontaktsprachen wie etwa das Slowenische war, lässt sich

6 Vgl. Pohl (2018). Viele deutschsprachige Endonyme in Kärnten und Osttirol, auch in der Steiermark (z. B. Graz), sind slowenischer Herkunft und manche slowenische Endonyme haben deutsche Wurzeln. Im Deutschen sind auch Lexemwörter slowenischer Herkunft präsent (vgl. Pohl 2019). Sie sind nicht nur in den Kontaktvarietäten wie Kärntner Mundarten zu finden, sondern auch im weiteren Großraum des Österreichisch-Bairischen sowie in der (regionalen) Umgangssprache in Österreich; in der regional gefärben Umgangssprache kommen auch syntaktische Interferenzen aus dem Slowenischen vor.

7 Zahlreiche deutsche Entlehnungen im Bereich der Lexik sind in allen sieben slowenischen Stammdialekten belegt. 
sowohl an Chronolekten, wie etwa in ihrer Phonologie (Šekli 2020), ablesen, als auch an den lexikalischen und syntaktischen Germanismen. Diese lassen sich - als Reflex puristischen Widerstandes unterschiedlicher Intensität - in verschiedenen diastratischen, diatopischen und diaphasischen Varietäten des Substandards ${ }^{8}$ ausmachen. Aus der Sicht des vorhin erwähnten gemeinsamen Kontaktareals sind sie zweifelsohne als Ergebnis andauernder und vielfältiger deutsch-slowenischer Kulturkontakte zu werten.

\section{DEUTSCHSPRACHIGE MINDERHEITEN IN SLOWENIEN UND IHR DEUTSCH}

\subsection{Diversität deutschsprachiger Siedler}

Neben der politisch bedingten Koexistenz beider Sprachen bis Ende des Ersten Weltkrieges (und während des Zweiten Weltkrieges zwischen 1941 und 1945) war Deutsch auch Muttersprache der Ansiedler im slowenischen ethnischen Raum, die zunächst vereinzelt seit dem 8. Jahrhundert und spätestens seit dem späten Mittelalter in der Krain wie auch in einigen Städten der Südsteiermark ansässig wurden. Mitten im slowenischen (einsprachigen) Gebiet lebend unterschieden sich die Angehörigen von

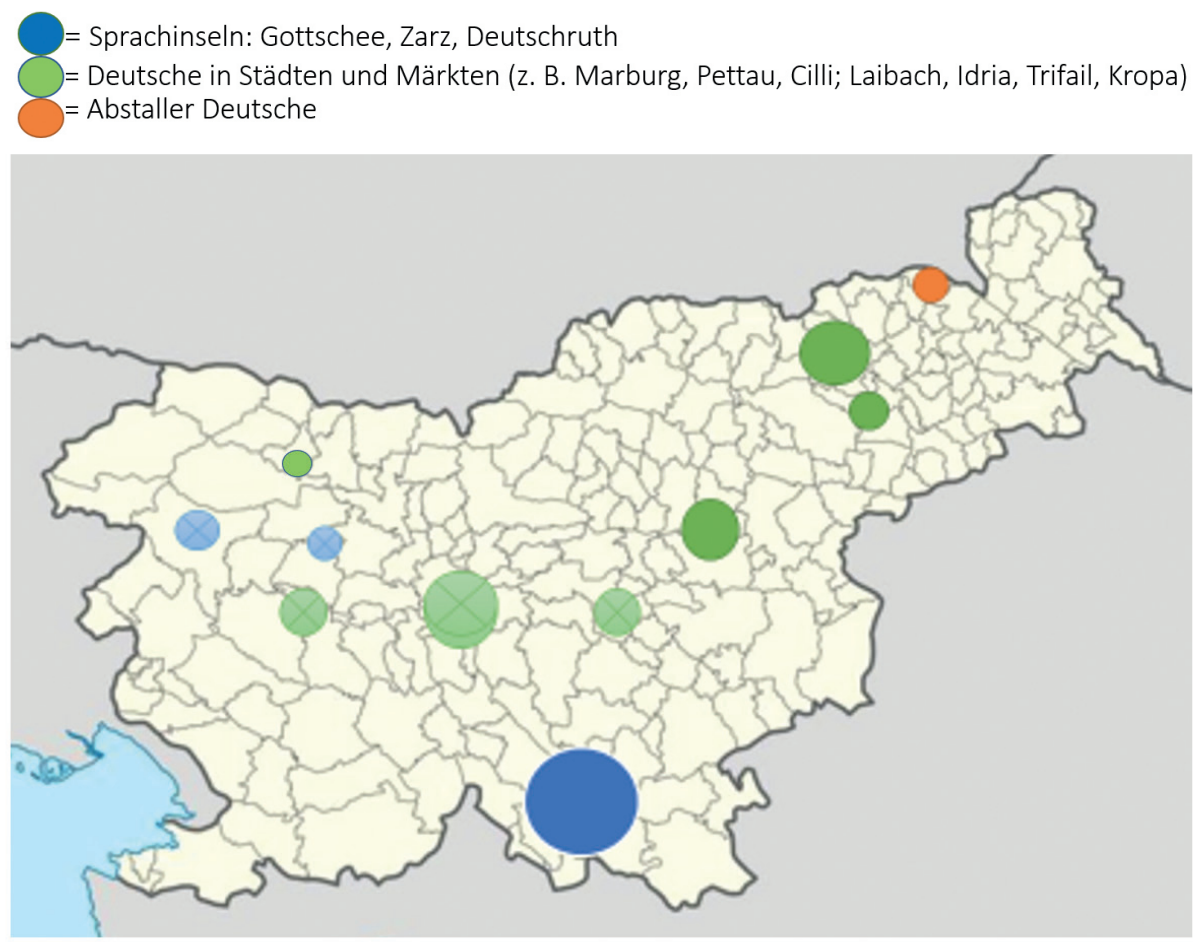

Abb. 2: Historische Verortung und Differenzierung der deutchen Mindheitern im slow. Sprachraum

8 Vgl. Krevs Birk (2017), Lipavic Oštir (2017), Javor Briški (2020). 
Minderheiten durch ihre soziale Provenienz: Geistliche (z. B. Škofja Loka/Bischofslack Kloster Stična/Sittich), Adelige und Großbesitzer mit ihren Verwaltern (z. B. Auersperger), Kolonisten (Sprachinseln Sorica/Zarz und Nemški Rovt/Deutschruth in der Oberkrain und Kočevska/Gottschee in der Unterkrain), Industrielle und Facharbeiter (z. B. in Kropa/Kropp, Idija/Idria, Domžale/Domschal), Beamte mit ihren Familien u. a. (z. B. in Maribor/Marburg an der Drau). Die Zarzer und Deutschruther Sprachinsel, die zu den ältesten deutschen Sprachinseln Europas zählen, wurden bis zu Beginn des 20. Jahrhunderts assimiliert. In Apače/Abstall gibt es eine Minderheit an der österreichisch-slowenischen Grenze seit der Grenzziehung nach dem Ersten Weltkrieg.

Die deutschsprachigen Gemeinschaften auf dem slowenischen ethnischen Gebiet (s. Abb. 1$)^{9}$ waren nicht nur sozial stark differenziert, sondern auch regional weit verteilt (in den urbanen Zentren wie etwa Laibach, Marburg a. d. Drau, Cilli, Pettau, Rudofswerth, Windisch Gräz; in kleineren Städten wie Krainburg, Idria, Kropp, Trifail, Windisch Feistriz usw.; im Abstaller Feld und anderen Gegenden wie Zarz, Deutschruth und Gottscheer Dörfer mit Gottschee als Zentrum ansässig). Auch demografisch gab es eine unterschiedliche Vertretung (bis 1918): entweder als nationale Mehrheit (z. B. in Marburg a. d. Drau, Gottscheer Sprachinsel) oder als Minderheit (z. B. in Laibach).

\subsection{Gottscheer Sprachinsel}

Das Schicksal der Sprachinsel Gottschee verlief anders als bwsp. in Zarz und wurde nicht durch Assimilierung, sondern durch Umsiedlung bestimmt. Die Gottscheer, die im 14. Jahrhundert im Süden der Unterkrain in eine recht karge Karstlandschaft mit kaum Transportwegen angesiedelt wurden, konnten bis in das 20. Jahrhundert hinein die Infrastruktur der insgesamt 176 Dörfer stärken und ein Regionalzentrum - die Stadt Gottschee/Kočevje - aufbauen und auch spätestens bis Ende des 19. Jahrhunderts ein gut funktionierendes Schulsystem wie auch kulturelle Einrichtungen etablieren (vgl. Ferenc 2005). Über sechs Jahrhunderte hindurch konnten sie ihr muttersprachliches Idiom, das Gottscheerische, eine auf dem Mittelhochdeutschen beruhende Mundart des Südbairischen, pflegen. Nach dem Ersten Weltkrieg konnte das Land keinen autonomen Status ${ }^{10}$ erlangen und wurde in den neu gegründeten Staat der Serben, Kroaten und Slowenen integriert. An die 12.000 Gottscheer lebten in der sprachlich weitgehend homogenen Sprachinsel Gottschee, und zwar bis zu ihrer Umsiedlung im Jahr 1941/1942 und anschließender Flucht aus dem Umsiedlungsgebiet über die slowenisch-österreichische Grenze im Jahr 1945. In den ersten Nachkriegsjahren waren sie weltweit zerstreut; sie wanderten vor allem nach Österreich, Deutschland, Kanada, in die USA und nach Australien aus, konnten jedoch trotz allmählich gut organisierter Netzwerke

9 Die Abb. 1. erstellt von U. K. B.

10 Im „Merkblatt zu den Friedensverhandlungen“ (verfasst vom Deutschen Volksrat für Gottschee, Oktober 1918) wurde der angestrebte Autonomiestatus für das „Gottscheerland“ u. a. mit der Bezugnahme auf seine Flächengröße und klare Grenzen, die Bevölkerungszahl, 600 Jahre lange Geschichte, politische Gliederung, Seelsorge, Finanzinstitute und Schulorganisation (von Kindergärten bis zum Obergymnasium) wie auch mit dem Verweis auf die verhältnismäßig kleineren Länder wie Monaco, San Marino, Lichtenstein und Andorra untermauert (vgl. Ferenc 2005: 60-61). 
ihre Muttersprache - Gottscheerisch - kaum pflegen. In der Gottschee wurden etwa fünf Prozent der Gottscheer nicht umgesiedelt, diese konnten aber Gottscheerisch im kommunistischen Land angesichts des Verbots wie auch der Verpönung des Deutschen und dadurch auch der Gottscheer Mundart lediglich im Familienkreis sprechen, was größtenteils zum Sprachwechsel und -bruch bei der nachfolgenden Generation führte.

\subsection{Gottschee und Siebenbürgen im Vergleich}

Die Existenz von im östlichen Europa verorteten Sprachminderheiten und eventuell Noch-Sprachinseln ist bei Weitem nicht selbstverständlich, wie dies die Situation in Slowenien veranschaulicht. Auch wenn sich die Sprachminderheiten über ihren Herkunftsraum geografisch einordnen und durch ihre Sprache bzw. Herkunftssprache (Deutsch) sowie aufgrund zusammenhängender Sprachenkonstellationen sprachlich bestimmen lassen, so zeigt sich jedoch oft eine Diskrepanz zwischen der globalen und regionalen Identitäts- und leider auch Existenzbewahrung auf kollektiver und individueller Ebene. Der Begriff der Identität muss dabei immer wieder relativiert werden: Obwohl sich die Identität aus diversen Perspektiven definieren lässt, ist sie aktuellen Diskursen zufolge jedenfalls sprachlich-kulturell, kollektiv oder individuell, prozessual und als solche provisorisch, polyvalent und unterschiedlich prägnant. Dies zeigt sich sowohl bei der historischen als auch aktuellen deutschsprachigen Minderheit in Schlesien, im Wolgagebiet, in Siebenbürgen, Tschechien, in der Gottschee, in Maribor/ Marburg a. d. Drau usw. Auf die gesamte Bandbreite der Problematik kann hier nicht ausführlich eingegangen werden. Obwohl gegenwärtig kollektiv oder individuell unternommene Bestrebungen und Bemühungen um Identitätswahrung und Spracherhalt auszumachen sind, die mehr oder minder erfolgreich verliefen, lässt sich die aktuelle Lage in diesem Areal eher als pessimistisch werten.

Zur Veranschaulichung seien im Folgenden Gottschee und Siebenbürgen als Fallbeispiel miteinander verglichen: In Siebenbürgen, einer Region mit einer bis zum Wendejahr 1989 stärksten deutschen Minderheit Rumäniens, gibt es heutzutage bis zu 30.000 rumäniendeutsche Muttersprachler (landesweit 36.000), was in Anbetracht der Zahl beispielsweise der Gottscheer im slowenischen Raum zunächst vielversprechend erscheint. Aber die Naivität, die Zahl als hoch einzuschätzen, ist plausibel und auch weitgehend bekannt, denn die Zahlen können auch täuschen: Bei den Siebenbürger Sachsen handelt es sich um weniger als zehn Prozent der Deutschstämmigen, die noch vor dem Zweiten Weltkrieg in Siebenbürgen ansässig waren. Angesichts der radikalen Abwanderung ${ }^{11}$ der Rumäniendeutschen ist die Frage nach dem Status des Deutschen als Muttersprache in Siebenbürgen oder Rumänien berechtigt. Entgegen des schwindenden Sprecheranteils wird Deutsch weiterhin nicht nur durch deutschsprachige

11 Vgl. Riehl (2007: 9-10): „Eine zweite Aussiedlungswelle ist seit Anfang der 1990er-Jahre zu beobachten. Durch die Öffnung der Grenzen und entsprechende Abkommen mit der deutschen Regierung vollzieht sich seit dieser Zeit eine radikale Abwanderung aus den Gebieten, in denen sich noch eine mehrheitlich deutschsprachige Bevölkerung befand. Davon betroffen sind Siebenbürgen und das Banat, aber das gilt auch für das gesamte Gebiet der ehemaligen Sowjetunion, d.h. die Ukraine und Russland.“ 
(Tages-)Zeitungen (z. B. Allgemeine Deutsche Zeitung für Rumänien) medialisiert, sondern auch als Unterrichtssprache bis zur tertiären Ausbildungsstufe ohne Unterbrechung eingesetzt (vgl. hierzu auch Sava 2020): „Die Entwicklung der Germanistik in Rumänien wurde vor und nach der politischen Wende (1989/1990) durch die deutsche Minderheit geprägt. Lehrende an deutschen Schulen in Rumänien waren bis zur Wende fast ausschließlich Angehörige der deutschen Minderheit.“(ebd.: 19).

Im offensichtlichen Gegensatz dazu lässt sich die Lage der Gottscheer erfassen. ${ }^{12}$ Bei den Gottscheern handelt es sich um eine Gemeinschaft weltweit zerstreuter Individuen, Familien oder auch Kulturvereine, die ihre Gottscheer Herkunft als Sprachinselidentität begreifen und sich

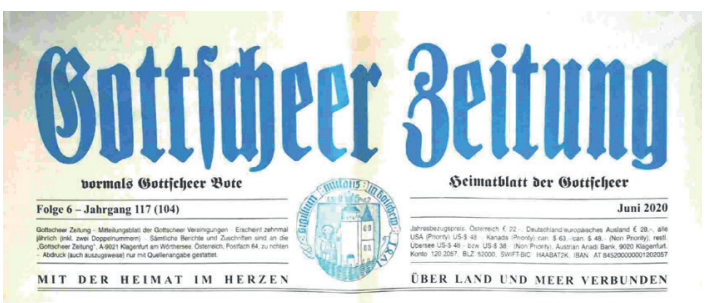

Das Heimatblatt für die Gottscheer in aller Welt

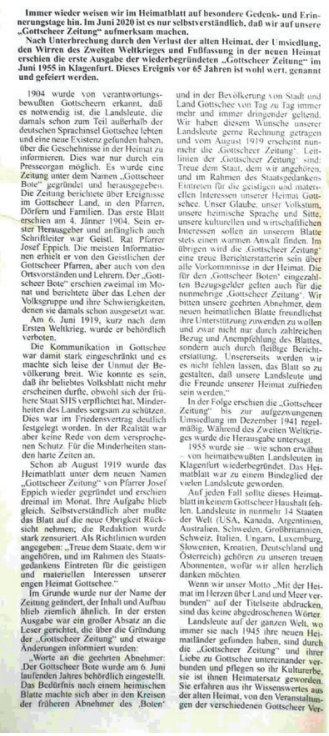

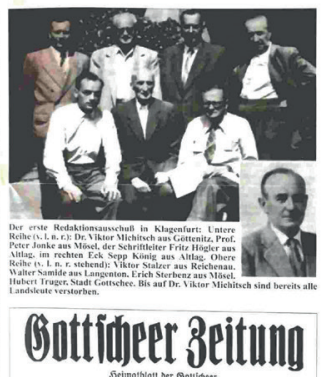
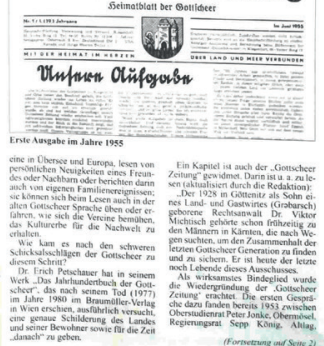
somit als Österreicher, Deutsche, Amerikaner, Kanadier, Australier, Slowenen u. a. definieren. Obwohl ihre Herkunftsregion Gottschee nicht mehr existiert, dient dieser Gemeinschaft das Gottscheerland weiterhin als Referenzrahmen für ihre Zugehörigkeit zur ehemaligen Sprachinsel. Sie organisieren Netzwerke und Vereine und versuchen, ihre Sprachinselherkunft und -kultur nach wie vor weiter zu pflegen und zu bewahren. Medial sind sie durch die in Klagenfurt erscheinenden Gottscheer Zeitung verbunden, das Presseorgan der ersten, zweiten, aber auch weiterer Generationen. ${ }^{13}$ Als „Heimatblatt für Gottscheer" führt das Blatt die weltweit zerstreuten Gottscheer zusammen, was durch den Slogan ,Mit der Heimat im Herzen über Land und Meer verbunden" (s. Abb. 1) aussagekräftig hervorgehoben wird:

Abb. 2: Titelblatt der Gottscheer Zeitung (Juni 2020), Jg. 117, Folge 6, Seite 1

12 Die Kontinuität des Schulunterrichts in deutscher Sprache wurde ab 1918 unterbrochen. Deutsche Schulklassen wurden nur unter strengster Einhaltung der Vorgaben für eine bestimmte Schüleranzahl zugelassen.

13 Die Gottscheer Zeitung erschien zunächst in Gottschee/Kočevje in den Jahren 1919-1941, ihre Vorgängerin war die Zeitung Gottscheer Bote. Von 1955 bis heute erscheint die Gottscheer Zeitung monatlich in Klagenfurt. 
Durch die Bezugnahme auf die ,alte Heimat“ beabsichtigt die Zeitung, zwischen den sich dieser Gemeinschaft als zugehörig empfindenden Individuen in der Diaspora zu vermitteln. Dies erfolgt durch Veröffentlichungen von individuell und kollektiv relevanten biografischen Erinnerungen an die ehemalige Sprachinsel als Heimatland wie auch durch Berichte über aktuelle Ereignisse aus den einzelnen Gottscheer Vereinen (z. B. aus Cleveland, Kitchener, Wien, Graz, Klagenfurt) sowie durch Ankündigung geplanter künftiger Aktivitäten. Für unterschiedliche Generationen der Gottscheer als primärer Adressatenkreis wird hierbei auf deren kulturelle und ethnische Herkunftsidentität referiert. Obschon das Gottscheerische als dialektale Varietät der ehemaligen Sprachinsel und - wie bekannt - inzwischen als beinahe untergegangene Muttersprache gilt, wird es als Elternidiom und erinnerte Muttersprache vermittelt. ${ }^{14} \mathrm{Zu}$ dieser weltweit verstreuten und durch ein traditionsreiches Presseorgan verbundenen Kultur- und Erinnerungsgemeinschaft gehören auch die in ihrer Heimatregion Gottschee/Kočevska verbliebenen Sprecherinnen und Sprecher - zur Zeit höchstens noch hundert ${ }^{15}-$, die im Laufe der Jahrzehnte nach dem Zweiten Weltkrieg im sozialistischen Slowenien vor schwerwiegende Herausforderungen gestellt waren. Diese ${ }^{16}$ versuchen heute - von Assimilation bedroht und/oder Resignation geplagt - vergeblich ihre Muttersprache Gottscheerisch zu bewahren.

Die hier eingebrachten Fallbeispiele, Gottschee und Siebenbürgen, sind unter dem Aspekt der verstärkten Schrumpfung der Sprachgemeinschaft ähnlich, da in beiden Fällen eine drastische Verminderung der Sprecherzahl offensichtlich ist. Allerdings können sie in ihren soziolinguistischen Spezifika kaum differenter ausfallen: In Siebenbürgen wird Deutsch als Muttersprache der Siebenbürger Sachsen von dem rumäniendeutschen Standard überdacht. Das Rumäniendeutsche (als der landesweit übliche Sprachgebrauch der in Rumänien lebenden Rumäniendeutschen) ist eine relativ homogene und überregionale Variante des Standarddeutschen bzw. eine von den Standardvarietäten des polyzentrischen Deutschen (vgl. Ammon 2015: LX). Im multiethnischen und mehrsprachigen Staat Rumänien hat Deutsch nach wie vor einen historisch gewachsenen Prestigestatus. Im Falle der Gottscheer (aber auch weiterer deutschsprachiger Gemeinschaften Sloweniens) ist eine Annäherung an das „Sloweniendeutsche“ keinesfalls möglich, weil dieses schlicht nicht existiert bzw. nicht existieren kann.

Allerdings kann festgestellt werden, dass die Bezeichnung „Sloweniendeutsche“ bereits Eingang in die Freie Enzyklopädie Wikipedia ${ }^{17}$ gefunden hat: Mit diesem Kom-

14 Dazu dient auch der Einbezug des Gottscheerischen in diverse Pressetexte der Gottscheer Zeitung. Als „binnendeutsche“ Presse (Földes 2019: 73, s. auch Anm. 10) scheint sie zugleich - trotz der Tradierung als Minderheitenzeitung - wenige saliente sprachliche Merkmale aufzuweisen.

15 Es gibt keine empirisch erhobenen Zahlen. Die Schätzung beruht auf Aussagen der Gottscheer (z. B. Maridi Tscherne) aus Pöllandl/Gottschee. Wie Jaklitsch (2019: 192, in Anl. an Hutter 1994) betont, sollte die Zahl der weltweit zerstreuten Gottscheer noch einige tausend betragen.

16 Um die Bewahrung des Gottscheerischen bemühen sich vereinzelt Vertreterinnen und Vertreter der jüngeren Generation, wie etwa Maridi Tscherne, die im letzten Jahrzehnt mehrere GottscheerischKurse organisierte.

17 Sloweniendeutsche. https://de.wikipedia.org/wiki/Sloweniendeutsche (12.09.2020). 
positum wird nämlich auf deutsche Muttersprachler als Altsiedler referiert - obwohl Slowenien als Staat erst seit 1991 existiert. Wie bekannt kann durch die Art der Medialisierung, abhängig von Akteuren, die Homogenisierung einer entstehungsgeschichtlich, soziostrukturell, sprachrepertoiredivers und regionalräumlich diversifizierten Minderheitenlandschaft erzielt werden. Die Bezeichnung „Sloweniendeutsche“ stellt einen Versuch der Homogenisierung von diversen deutschsprachigen Sprachgemeinschaften in Slowenien dar, die sozial, regional und sprachlich unterschiedlich verortet sind. Dies verdeutlicht auch ein Vergleich zwischen der deutschen städtischen Bevölkerung Ljubljanas/Laibachs oder Maribors/Marburgs und den Gottscheern: Bei den Gottscheern handelte es sich um eine weitgehend rurale Bevölkerung in einer abgelegenen Region im Süden der Unterkrain, wo einige Angehörige der Sprachgemeinschaft und ihre Nachfahren nach wie vor leben. Das Gottscheerische ist eine kollektiv und individuell erinnerte und noch gegenwärtig gesprochene muttersprachliche Varietät, die die wenigen Sprecher der Vorkriegsgeneration ${ }^{18}$ und einige ihre Nachfahren ${ }^{19}$ in Slowenien in ihrem Umgang miteinander sozial- und situativbedingt gebrauchen.

\section{DEUTSCHSPRACHIGE MINDERHEITEN SLOWENIENS IM MEDIALEN DISKURS}

Auf den Status der deutschen Sprache und Kultur in Konstellation mit weiteren Sprachen/Kulturen verweist das materielle und immaterielle Kulturererbe der deutschen Sprachgemeinschaften, ihr Kollektivgedächtnis und das von ihnen geprägte Werte- und Symbolsystem. Sprach- und Kulturkontaktprozesse und insbesondere Migrationen, von denen diese Minderheitengruppen betroffen waren und nach wie vor sind, schlagen sich in vielfältigen, die sprachliche und kulturelle Identität betreffenden Phänomenen nieder. Sie verweisen auf heterogene und homogene sprachlich-kulturelle Erscheinungsformen und reichen von unterschiedlich stark ausgeprägter äußerer und innerer Mehrsprachigkeit bis hin zu gedächtniskultureller Diversifizierung oder Homogenisierung, die aufgrund praxisbedingter sprachlich-kultureller Verschiebungen ihren Ausdruck in Multimedia finden.

Ein Beispiel dafür findet sich auf der Internetseite der Österreichischen Botschaft Laibach, in der Rubrik Deutschsprachige Volksgruppe in Slowenien ${ }^{20}$, wo Angaben zu dem Lebensraum der ,autochthonen deutschsprachigen Volksgruppe“ geboten werden: „Heute

18 Das Gottscheer Deutsch wird - sozialsituativbedingt - auch noch von den Gottscheern der Vorkriegsgeneration gesprochen, die außerhalb Sloweniens (z. B. in Kärnten, den USA, Kanada) leben. Dies veranschaulicht auch der Dokumentarfilm „Gottscheabar Lont. Das verlorene Kulturerbe“ (Hermann Leustik, Uroš Zavodnik, 2015), in dem der Hauptdarsteller Frank Mausser (wohnhaft in Klagenfurt, geb. 1938 in Altlag/Stari Log, s. auch Mausser/Krevs Birk 2017) ausschließlich Gottscheerisch gebraucht.

19 Neben gottscheerischen Sprachkursen gab Maridi Tscherne im letzten Jahrzehnt auch das gottscheerisch-slowenische Wörterbuch (2012) sowie einige literarische Texte auf Slowenisch, Deutsch und Gottscheerisch heraus.

20 https://www.bmeia.gv.at/oeb-laibach/oesterreich-in-slowenien/deutschsprachige-volksgruppe-inslowenien/ (30.09.2020). 
leben sie vor allem in der ehemaligen Untersteiermark, der heutigen Region Štajerska, in Städten wie Maribor/Marburg, Celje/Cilli an der Sann, Ptuj/Pettau oder Apače/Abstall, im Südosten des Landes in der Gottschee in Siedlungen, die zu den Gemeinden Kočevje, Dolenjske Toplice und Semič gehören, sowie in Laibach." ${ }^{\text {21 }}$ Die genannten Orte, einst historische Wohnorte der deutschen Sprachgemeinschaften, standen, wie bereits erwähnt, in der k. u. k. Monarchie keinesfalls in engerem Kontakt, da sie - trotz der Rolle des Deutschen als Dachsprache - aufgrund der regionalen Entfernung (z. B. Laibach Marburg a. d. D. - Gottschee), durch soziale Differenzen (urbane vs. rurale Gegend: z. B. Marburg vs. Gottschee) und etlicher Unterschiede im Sprachrepertoire (z. B. Laibacher Krainer Gemeinsprache ${ }^{22}$ - Marburger Deutsch ${ }^{23}$ - Südsteierisch - Gottscheerisch ${ }^{24}$ ) wenig überregional miteinander vernetzt waren, allerdings als intraregionale Netzwerke (z. B. innerhalb der Gottscheer Sprachinsel, Südsteiermark, Laibach und Umgebung) fungierten und zu ihren regionalen urbanen Zentren tendierten. In diesen Orten konnten nach der Selbstständigkeitserklärung Sloweniens (1991) mehrere Kulturvereine ${ }^{25}$ entstehen, die dem „Dachverband der Kulturvereine der deutschsprachigen Volksgruppe in Slowenien/Zveza kulturnih društev nemško govoreče narodne skupnosti v Sloveniji“" angeschlossen sind. Auf der Webseite wird auch die homogenisierende Funktion dieses Dachverbandes angeführt, nämlich die unterschiedlichen Interessen dieser Kulturvereine zu vertreten, die unterschiedliche Schwerpunkte bei ihren Aktivitäten setzen. Im weiteren Text wird die Existenz eines weiteren Dachverbandes, nämlich des „Dachverbandes der Gottscheer Organisationen“ (durch „,sogenannten“" attribuiert), keinesfalls ausgeblendet, im Gegenteil, sie wird ausdrücklich erwähnt, jedoch werden die Mitgliederanzahl wie auch seine Aktivitäten nicht angeführt. Aus mindestens formalen Gründen wird seine Funktion in Frage gestellt, da er „ohne Kulturvereine als Mitglieder“ fungiere. Durch die Medialisierung der Divergenz der Dachvereine wird eine Positionierung durch eine inhaltliche Ausdifferenzierung geäußert. Somit wird zugleich ein Bild der weitgehenden Einheitlichkeit der Vereine im „Dachverband der Kulturvereine der deutschsprachigen Volksgruppe in Slowenien“ vermittelt, das beim Anliegen der Volksgruppe „um verfassungsrechtliche Anerkennung und Erhalt ihres kulturellen Erbes“ dringend benötigt wird.

21 Hervorhebung im Original.

22 Javor Briški (2012: 591; 2020: 189), Krevs Birk (2019: 157).

23 Jesenšek (2019: 178).

24 Tscherne (2012), Krevs Birk (2019: 160).

25 Auf der Internetseite der Österreichischen Botschaft Laibach werden sieben Kulturvereine genannt. Dem Dachverband gehören folgende Kulturvereine an: Verein Freiheitsbrücke Marburg/Društvo Most svobode Maribor, Kulturverein deutschsprachiger Frauen Brücken/Kulturno društvo nemško govorečih žena Mostovi Maribor, Kulturverein Cilli an der Sann/Kulturno društvo Celje ob Savinji, Gottscheer Altsiedler Verein Krapflern/Društvo Kočevarjev staroselcev Občice, Kulturverein deutschsprachiger Jugend (KDJ) in Laibach/Društvo nemško govoreče mladine v Ljubljani, Gottscheer Verein Laibach/Etnološko kočevarsko društvo Ljubljana, Kulturverein Abstaller Feld/ Kulturno društvo Apaško Polje. Vgl. hierzu https://www.bmeia.gv.at/oeb-laibach/oesterreich-inslowenien/deutschsprachige-volksgruppe-in-slowenien/ (30.09.2020). 


\section{ZUM SCHLUSS: DIE NEUE LAIBACHER ZEITUNG}

Abschließend soll ein aktueller Fall der Zusammenführung und Homogenisierung von Altsiedlern in Slowenien (in der Krain, Steiermark) und ihren Nachfahren am Beispiel der seit 2017 erscheindenen Laibacher Zeitung erwähnt werden. Die seit drei Jahren herausgegebene zweisprachige Zeitung fällt insbesondere durch ihren Namen auf, da sie denselben Namen trägt wie die bereits vor mehr als 200 Jahren erschienene Laibacher Zeitung. Die historische Laibacher Zeitung (1784-1918) wird als Inbegriff der Präsenz und Existenz der Deutschstämmigen sowie der Einflussnahme der vermittelten deutschen Kultur $^{26}$ auf die slowenische Umgebung bis Ende des Ersten Weltkrieges aufgefasst. Sie war das einflussreichste und am längsten erscheinende deutsche Periodikum der Krain, ${ }^{27}$ zugleich auch das führende deutschsprachige Presseorgan im slowenischen ethnischen Gebiet in der Habsburgerzeit.

Das moderne Periodikum des „Dachverbandes der Kulturvereine der deutschsprachigen Volksgruppe " in Slowenien übernimmt bei der Ersterscheinung 2017 von seiner traditionsreichen Vorgängerin deren Namen Laibacher Zeitung. Da Sachnamen wie Buchtitel und Periodika nicht nur eine Benennungs-, sondern auch eine Identifikationsfunktion haben, ist mit der Wahl des Namens Laibacher Zeitung, auch in seiner typografischen Form, ein Zeichen gesetzt worden. Der Name hat hierbei eine hohe Relevanz für die potenziellen Adressaten, wobei der zweisprachige Untertitel „Unabhängige Zeitung für Volksgruppe, Wissenschaft und Kultur/Samostojni časopis za narodno skupnost, znanost in kulturo " an die zweisprachige deutsch-slowenische Identität der Leserschaft appelliert. Darüber hinaus lässt der Titel konkrete Ambitionen erkennen. Die (potenzielle) Leserschaft wird dabei folgendermaßen angesprochen: „Auf Initiative des Kulturvereins der deutschsprachigen Jugend wurde die Zeitung im Jahr 2017 wiederbelebt und erscheint vierteljährlich mit den aktuellen Berichten und Themen zur allgemeinen Lage der deutschsprachigen Volksgruppe in Slowenien." Die Herausgabe der Zeitung, die zunächst gedruckt erschienen ist und seit April 2020 digital, ${ }^{28}$ wird als eines der wichtigsten Ereignisse in den letzten drei Jahrzehnten seit der Selbstständigkeitserklärung Sloweniens präsentiert, was für die Zukunft der deutschen Minderheit in Slowenien von Bedeutung sein könnte. Durch dieses Periodikum (bis zu diesem Zeitpunkt sind acht Ausgaben erschienen, s. Abb. 3) werden die deutschen Altsiedler und

26 Da auch damals keine völlig homogene deutsche Kultur existierte, muss der Begriff „Kultur“ der aktuellen Diskussionen zufolge auch als Konstrukt verstanden werden.

27 Erstmals erschien die Laibacher Zeitung am 1. Januar 1784 unter der Redaktion von Kleinmayr, einem in Klagenfurt wirkenden Drucker, Verleger und Buchhändler. Das Blatt enthielt unterschiedliche Beilagen, darunter auch das Amtsblatt. Im Laufe der Jahrzehnte wurde die Zeitung mit den Beilagen Laibacher Wochenblatt, Blätter aus Krain, Illustriertes Unterhaltungsblatt und Illyrisches Blatt herausgegeben. 1784 wurde die Zeitung um eine Inseratenbeilage (Wöchentliches Kundschaftsblatt und Inseratblättchen) erweitert (vgl. Birk 2000). Seit der Aufklärungszeit bis in die 1. Hälfte des 19. Jahrhunderts war die Laibacher Zeitung an der Etablierung des slowenischen Kulturfeldes maßgeblich beteiligt (vgl. Birk/Nicklas/Kramberger/Urekar Osvald/Zver 2020: 219ff.).

28 https://laibacher-zeitung.si (01.05.2020), Chefredakteur Christian Lautischer. 
ihre Nachfahren, die einzelnen Altsiedler mit Noch-Erst- und Zweitsprache Deutsch, die in den urbanen Zentren Sloweniens (Ljubljana/Laibach, Maribor/Marburg a. d. Drau, Celje/Cilli u. a.) wie auch in weiteren Provinzen (Kočevska/die Gottschee, Apače/Abstall) zerstreut sind, zusammengeführt.

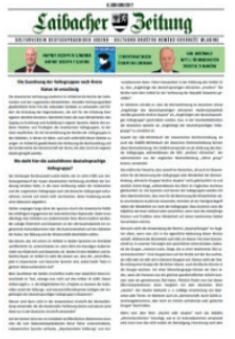

Saibacfier Beitung

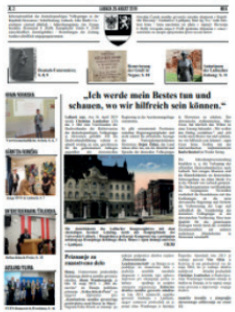

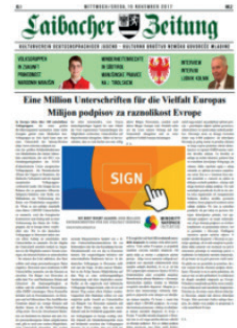

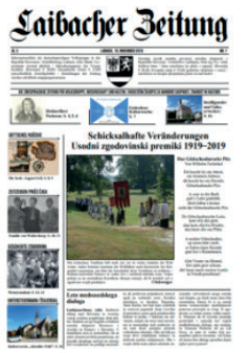

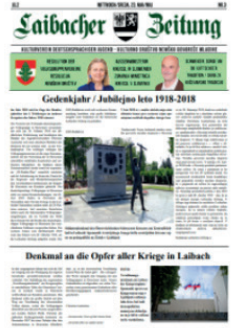

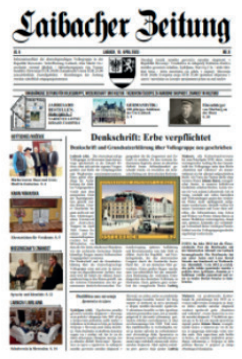

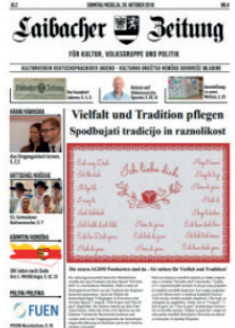
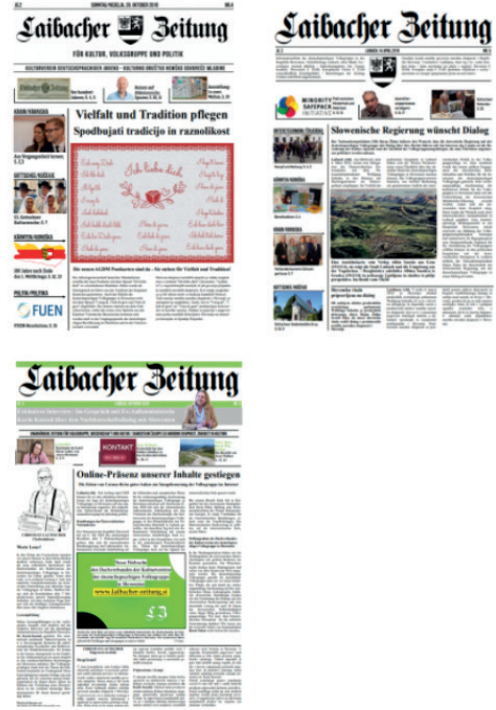

Abb. 3: Die Ausgaben der Laibacher Zeitung auf der Webseite des Periodikums ${ }^{29}$ (https://laibacher-zeitung.si/o-casopisu/)

Auf die deutsch-slowenische Zweisprachigkeit des Lesepublikums wird größtenteils durch zweisprachige (gelegentlich auch englischsprachige) Texte referiert, obwohl die Frage, ob dieser Homogenisierungsversuch sämtliche Altsiedler erreicht, offen bleibt: „Der Dachverband der Kulturvereine der deutschsprachigen Volksgruppe in Slowenien wird aus 7 Vereinen mit mehr als 1.500 Mitgliedern gebildet. " ${ }^{\text {"30 }}$ Das Medium etabliert sich als eine gemeinsame Plattform für die zivilgesellschaftliche Repräsentation. Durch die Medialisierung der auf die deutsche Sprachgemeinschaft bezogenen Gegebenheiten wird u. a. der Anspruch (unter anderem auch seitens der Republik Österreich unterstützt) auf die verfassungsrechtliche Anerkennung der deutschen Sprachminderheit in Slowenien erhoben - ein Status, der der italienischen und ungarischen Minderheit zuerkannt ist und der bis dato für die deutsche Sprachgemeinschaft in Slowenien ausgeblieben ist.

29 Die acht Ausgaben der Laibacher Zeitung sind auf der Webseite des Periodikums präsentiert: https://laibacher-zeitung.si/o-casopisu/ (28.11.2020).

30 https://laibacher-zeitung.si/kdo-smo/(15.07.2020). 


\section{Literatur}

AMMON, Ulrich (2015) Die Stellung der deutschen Sprache in der Welt. Berlin/München/Boston: de Gruyter.

BIRK, Matjaž (2000) ,,--- vaterländisches Interesse, Wissenschaft, Unterhaltung und Belehrung ---“. Illyrisches Blatt (Ljubljana, 1819-1849), literarni časopis v nemškem jeziku v slovenski provinci predmarčne Avstrije. Maribor: Slavistično društvo.

BIRK, Matjaž/Thomas, NICKLAS/Petra, KRAMBERGER/Anja, UREKAR OSVALD/Sašo, ZVER (2020) „Transfer der Aufklärung in der deutschsprachigen Presse im Elsass und in der Krain im späten 18. Jahrhundert." Historisches Jahrbuch, 2020, 140, 216-239.

Forschungszentrum Deutsch in Mittel-, Ost- und Südosteuropa. Aufgaben und Ziele. https://www.uni-regensburg.de/forschung/dimos/startseite/index.html (15.05.2020).

FERENC, Mitja (2005) Kočevska pusta in prazna: nemško jezikovno območje na Kočevskem po odselitvi Nemcev. Ljubljana: Modrijan.

FERENC, Mitja/Joachim HÖSLER (Hrsg.) (2011) Spurensuche in der Gottschee. Deutschsprachige Siedler in Slowenien. Potsdam: Deutsches Kulturforum östliches Europa.

FÖLDES, Csaba (2011) „Mitteleuropa als Erkenntniskategorie und Raum-Modell: ein Arbeitsfeld für die germanistische Kontaktlinguistik." In: Z. Glovacki-Bernardi (Hrsg.), Deutsch in Südost- und Mitteleuropa. Kommunikationsparadigmen im Wandel. Zagreb: FF press, 7-25.

FÖLDES, Csaba (2019) „Die Deutsche Allgemeine Zeitung. Anmerkungen zur Sprache der kasachstandeutschen Presse." Linguistica 59/1, 71-97.

FOUCAULT, Michel (2008) Die Hauptwerke. Frankfurt a. Main: Suhrkamp.

Gottscheer Zeitung (2020). Titelseite (Juni 2020), 117, 6, 1.

INFOPORTAL ÖSTLICHES EUROPA. https://osteuropa.lpb-bw.de/ (12.09.2020).

JAKLITSCH, Helena (2019) „Kočevarščina oz. kočevska nemščina, jezik Kočevarjev oz. kočevskih Nemcev.“ In: M. Šekli/L. Rezoničnik (Hrsg.), 186-194.

JAVOR BRIŠKI, Marija (2012) „Slowenien.“"In: A. Greule/J. Meier/A. Ziegler (Hrsg.): Kanzleisprachenforschung: ein internationales Handbuch. Berlin/New York: de Gruyter, 589-598.

JAVOR BRIŠKI, Marija (2020) Nemščina v diahroni perspektivi. Ljubljana: Znanstvena založba Filozofske fakultete.

JESENŠEK, Vida (2019) „Nemščina v Mariboru: preplet kulturnozgodovinskih in jezikovnih danosti.“ In: M. Šekli, L. Rezoničnik (Hrsg.), 178-185.

KREVS BIIRK, Uršula (2017) „Deutsche und slowenische Toponymika mit Blick auf Ortsnamen außerhalb des geschlossenen deutschen und slowenischen Sprachraums." Germanistische Beiträge 41, 115-133.

KREVS BIRK, Uršula (2019), Zu einigen Aspekten des Deutschen als Kontaktsprache des Slowenischen." Linguistica 59/1, 155-174.

KURZOVÁ, Helena (1996) „Mitteleuropa als Sprachareal.“ Acta Universitatis Carolinae - Philologica 5, Germanistica Pragensia XIII, 57-73.

LAIBACHER ZEITUNG. https://laibacher-zeitung.si/ (01.06.2020). 
LIPAVIC OŠTIR, Alja (2017) Substandardsprachliche Germanismen im Slowenischen. Wien: LIT.

MAUSSER, Frankie/Uršula KREVS BIRK (2017) Sprachbiographie anhand eines Fragenkatalogs. Manuscript. Klagenfurt: Privatarchiv.

MEIER, Jörg (Hrsg.) (2018) Jahrbuch des Bundesinstituts für Kultur und Geschichte der Deutschen im östlichen Europa. Sprache. Band 26. Oldenburg: de Gruyter.

MEMIĆ, Nedad (2019) „Besonderheiten der deutschen Verwaltungssprache im k. u. k. Bosnien-Herzegowina." Linguistica 59/1, 187-185.

MÜLLER, Jakob (2014) Kočevar ima samo eno domovino/Göttscheabar hot lai oin Hoimöt. Grosuplje: KD sv. Mihaela.

NEWERKLA, Stefan Michael (2017) „Kontaktareale in Mitteleuropa am Beispiel Altösterreich.“'In: Ch. Mauerer (Hrsg.), Mehrsprachigkeit in Mittel-, Ost, und Südosteuropa. Gewachsene historische Vielfalt oder belastendes Erbe der Vergangenheit. Regensburg: Pustet, 17-32.

POHL, Heinz-Dieter (2018). Sprachen und Sprachinseln im südalpinen Raum - ein Überblick. http://members.chello.at/heinz.pohl/Sprachinseln.htm (30.08.2020).

POHL, Heinz-Dieter (2019) „Deutsch-slowenische Wechselbeziehungen in der Sprache der traditionellen Kärntner Küche.“ Linguistica 59/1, 253-265.

RIEHL, Claudia Maria (2007) „Die deutschen Sprachgebiete in Mittel- und Osteuropa." In: L. M. Eichinger/A. Plewnia/C. M. Riehl (Hrsg.), Handbuch der deutschen Sprachminderheiten in Mittel- und Osteuropa. Tübingen: Gunter Narr, 1-16.

SAVA, Doris (2020) „Heiter bis wolkig. Zum 50. Jubiläum des Hermannstädter Lehrstuhls für Germanistik." Germanistische Beiträge 46, 15-48.

SCHEURINGER, Hermann (2017) „Historische Mehrsprachigkeit mit Deutsch im östlichen Europa." Germanistische Beiträge 39, 187-203.

„SLOWENIENDEUTSCHE. “ Wikipedia. Die freie Enzyklopädie. https://de.wikipedia.org/wiki/Sloweniendeutsche (12.09.2020).

ŠEKLI, Matej (2015) „Semantische Felder der deutschen Lehnwörter im Slowenischen." Slavica Tergestina 16, 144-168.

ŠEKLI, Matej (2019) „Spoznaj svoj jezik in jezik svojega soseda.“ In: M. Šekli, M./L. Rezoničnik, L. (Hrsg.), 9-12.

ŠEKLI, Matej/Lidija REZONIČNIK (Hrsg.) (2019). Slovenski jezik in njegovi sosedje. Ljubljana: Zveza društev Slavistično društvo Slovenije.

ŠEKLI, Matej (2020) „Relativna in absolutna kronologija (bavarsko) staro- in srednjevisokonemških izposojenk v slovenščini.“ Jezikoslovni zapiski 26, 1. 7-25.

TSCHERNE, Maridi (2012) Kočevarsko-slovenski slovar. Koprivnik/Nesseltal: Zavod za ohranitev kulturne dediščine Nesseltal Koprivnik/Einrichtung für die Erhaltung des Kulturerbes Nesseltal.

Verlorene Gottscheer Dörfer/Izgubljene kočevarske vasi. http://kocevarskevasi.si/de (20.09.2020).

ZAVODNIK, Uroš (2015) Gottscheabar Lont. Das verlorene Kulturerbe/ Dežela Kočevarjev. Izgubljena kulturna dediščina. Ein Dokumentarfilm. Regie: U. Zavodnik/H. Leustik. Klagenfurt/Wien/Graz: Gottscheer Landsmannschaft/Institut für Medien- und Kommunikationswissenschaft. 


\section{Zusammenfassung \\ ÜBERLEGUNGEN ZU DISKURSIVEN PRAXEN ÜBER ÖSTLICHES EUROPA UND DEUTSCHSPRACHIGE MINDERHEITEN IM SLOWENISCHEN RAUM}

Im östlichen Raum als einem Areal, wo über Jahrhunderte hinweg Migrationsbewegungen und Mehrsprachigkeit bestimmend waren, nahm die deutsche Sprache eine bedeutende Rolle wahr - sei es als Kontaktsprache in diversen dynamischen Konstellationen zu koexistenten (Nachbar-)Sprachen oder als Sprache unterschiedlicher deutschsprachiger Siedlergruppen. Hierbei erweist sich das Syntagma östliches Europa als Deiktikon, was u. a. durch mehrere Übersetzungsäquivalente im Slowenischen offensichtlich ist. Das im östlichen Europa polyfunktionale Deutsch - als historisch bedingte dominante Sprache im Habsburgerreich und gegenwärtig interregionale Sprache mit Vermittlungsfunktion - bezieht diesen Status durch seine konstitutive Teilhabe am mitteleuropäischen Sprachareal, zu dessen Kontaktareal Altösterreich, die österreichische Standardvarietät und das Ungarische, Tschechische, Slowakische, das Polnische und Slowenische wie auch das Kroatische, das im ehemaligen Gebiet der österreichisch-ungarischen Monarchie gesprochene Serbische und Bosnische, das Rumänische und Ukrainische gehören. Unter diesem Aspekt überschneidet sich das östliche Europa stark mit Mitteleuropa.

Vor dem Hintergrund der mittel- und osteuropäischen Verortung des slowenischen Raums wird im Beitrag Deutsch als dominante Kontaktsprache wie auch Muttersprache diverser Siedler und ihrer Nachfahren erfasst. Im Mittelpunkt der Betrachtungen stehen dabei die Gottscheer, deren muttersprachliches Idiom allerdings stark gefährdet ist, jedoch noch gesprochen wird. Der Vergleich mit der Lage der Deutschstämmigen in Siebenbürgen (Rumänien) dient hierbei der Differenzierung soziolinguistischer Charakteristika der Sprachgemeinschaft der Gottscheer. Aus aktueller Sicht werden exemplarisch auch einige diskursive Praxen und Ziele wichtiger Akteure mit Blick auf die Gottscheer und andere deutschsprachige Gruppen in Slowenien aufgezeigt.

Schlüsselwörter: östliches Europa, mitteleuropäisches Kontaktareal, Kontaktareal Altösterreich, deutsche Sprachminderheiten, Siebenbürger Sachsen, Gottscheer

\section{Abstract \\ THE DISCOURSE ON GERMAN IN EASTERN EUROPE AND THE GERMAN-LANGUAGE MINORITIES IN SLOVENIA}

Eastern Europe is an area with a millennia-long history of migrations and multilingualism where the German language had an important function as a contact language, which was involved in intensive and dynamic interactions with its neighbouring or co-exististing languages. It was often also the language of immigrants who settled in the area. The paper discusses the term östliches Europa, which partially overlaps with 
that of Central Europe and which has a deictic force that is reflected in its various Slovene translation equivalents. As a dominant language in the past and an interregional language at present, German has preserved its importance in Central Europe in general. Part of Central Europe is also the linguistically significant Old Austrian Contact Area that includes the Austrian variety of standard German, Polish, Czech, Slovak, Hungarian, Slovene as well as Croatian, Serbian and Bosnian, Romanian and Ukrainian.

In view of Slovenia being an integral part of Central and Eastern Europe, the article tries to give an outline of the presence of German in the Slovene-speaking area, both as a contact language which had a great impact on Slovene itself as well as first language of immigrants who arrived there many centuries ago. Special attention is given to the Gottscheer Germans whose language is nowadays still spoken, although it is endangered. The situation of the Gottscheer Germans is compared to that of the Germans from Transylvania (Romania). Highlighted are some sociolinguistic features as well as some discursive practices in the media typical of different agents who contribute to the status construction of Gottscheer Germans and their language variety.

Keywords: Eastern Europe, Central European linguistic area, Old Austrian contact area, German language minorities, Transylvania Germans, Gottscheer Germans

\section{Povzetek \\ O DISKURZU O NEMŠČINI V VZHODNOEVROPSKIH REGIJAH TER NEM- ŠKIH JEZIKOVNIH MANJŠINAH V SLOVENSKEM PROSTORU}

Vzhodnoevropske regije so prostor večstoletnih migracij in večjezičnosti, $v$ katerem je nemški jezik imel pomembno vlogo, bodisi kot stični jezik, ki je bil v različnih intenzivnih in dinamičnih odnosih s svojimi sosedi ali celo sobivajočimi jeziki, bodisi kot jezik priseljencev izpred več stoletij. V članku je obravnavana sintagma vzhodna Evropa (östliches Europa), ki se v germanističnem pogledu izkaže za deiktično, kar se pokaže denimo spričo prevodnih ustreznic $\mathrm{v}$ slovenščini. Znotraj polja funkcij nemščine kot dominantnega jezika $\mathrm{v}$ preteklosti in medregionalnega jezika s posredniško vlogo $\mathrm{v}$ sedanjosti je nemščina konstitutivni jezik srednjeevropskega jezikovnega areala, del tega pa je tudi t. i. staroavstrijski stični areal, ki skupaj z avstrijsko standardno različico nemščine zajema poljščino, češčino, slovaščino, madžarščino, slovenščino, poleg tega pa tudi hrvaščino, srbščino in bosanščino v času Avstroogrske ter celo romunščino in ukrajinščino. V tem oziru se razumevanje sintagme östliches Europa deloma prekriva s srednjo Evropo.

$\mathrm{Z}$ ozirom na vpetost $\mathrm{v}$ srednjo- in vzhodnoevropsko regijo je $\mathrm{v}$ prispevku prikazana prisotnost nemščine na slovenskih tleh - nemščina ni bila le stični jezik, ki je pustil sledi v več različnih segmentih slovenščine, temveč tudi jezik rojenih govorcev, priseljencev izpred več stoletij. V prispevku so najbolj izpostavljeni Kočevarji, katerih jezik je močno ogrožen, a še vedno v rabi. Ob primerjavi z romunskimi Nemci na 
Sedmograškem so izpostavljene njihove sociolingvistične značilnosti ter tudi nekatere diskurzivne prakse v medijih različnih akterjev, ki trenutno konstruirajo položaj Kočevarjev in kočevarščine ter nemške manjšine in nemščine v Sloveniji.

Ključne besede: vzhodna Evropa, srednjeevropski jezikovni areal, staroavstrijski stični areal, nemške jezikovne manjšine, sedmograški Nemci, Kočevarji 